\title{
Radiation Detectors for Medical Applications
}

\author{
S. Tavernier, A. Gektin, B. Grinyov, and W.W. Moses
}

New York, NY: Springer, 2006, 315 pages, $\$ 169$

This book is the proceedings of the 2005 NATO Advanced Research Workshop on Radiation Detectors for Medical Application that was held in Alushta, Crimea, Ukraine, on September 19-23 of 2005 and is published as part of the NATO Security Through Science Series. The spirit of such NATO workshops is to give an overview of the present state of a specific field rather than to bring the latest research results. In this regard, the book provides an overview of radiation detectors for medical applications in general, with particular emphasis on scintillation detectors.

The book is composed of a collection of 13 articles from 14 keynote speakers. The first 4 chapters contain a general overview of the applications of radiation detectors in medicine, with specific applications to CT (chapter 2), $\gamma$-ray imaging and SPECT (chapter 3), and PET (chapter 4). Chapter 5 is devoted to semiconductor detectors and their use for quality assurance in radiotherapy applications, including integral dosimetry in photon, electron, and neutron therapies; microdosimetry in hadron therapy; and mini dosimetry in synchrotron therapy. A discussion of semiconductor detectors for portal imaging with medical linear particle accelerators is also included in this chapter.

Chapters 6 and 7 are devoted to the ClearPET small-animal scanner (raytest USA, Inc.), which is the result of the Crystal Clear project, an international collaboration of research institutes working to develop new generations of scanners for PET. Chapter 6 focuses on the design considerations of this scanner and its performance characterization; whereas chapter 7 presents details on the lutetium yttrium silicon dioxide (LYSO)/lutetium yttrium aluminum perovskite (LuYAP) phoswich detector and crystal identification methods used in the ClearPET scanner design.

The remaining 6 chapters (chapters 8-13) are devoted to providing an overview of current research in scintillation detectors and their use in medical imaging. For example, chapter 8 provides an overview of desirable scintillator attributes and their fundamental limitations with respect to speed, light output, energy resolution, and density. Specific attention is given to $\mathrm{Ce}^{3+}$-activated scintillators for $\mathrm{x}$-ray and $\gamma$-ray imaging. Chapter 9 focuses on the materials, designs, and light collection conditions of scintillation detectors in general. Chapter 10 discusses the current and future use of lutetium oxyorthosilicate:Ce scintillators in PET, whereas chapter 11 covers other inorganic scintillators in PET, such as LuYAP, LYSO, lanthanum chloride: $\mathrm{Ce}$, lanthanum bromide: $\mathrm{Ce}$, and lutetium iodide:Ce. Chapter 12 deals with the manufacturing and growth processes of scintillation detectors. Finally, chapter 13 focuses on the nonproportionality and energy resolution of scintillation detectors and their impact on intrinsic resolution.

All chapters include figures, tables, and illustrations that have been reproduced with good quality. Each chapter follows the format of a journal paper, complete with an abstract, introduction, methods, and conclusion sections. In addition, all chapters end with a comprehensive list of references that I found to be useful for additional reading on this topic.

One limitation is redundancy in some of the material in the last 6 chapters (chapters 8-13). This, however, is partly expected, especially in proceeding books for conferences at which speakers are unaware of what others will be focusing on during their talks. I suspect that was the reason behind combining 2 keynote talks into 1 chapter as indicated in the book preface. Notwithstanding, I found some of the redundancy to be helpful in reinforcing my understanding of scintillation detectors and their performance characteristics.

Overall, I found the book to be a valuable resource on scintillation radiation detectors and their medical applications. This book should be part of any medical physics, health physics, radiology, or radiation oncology library. It will also be a good reference for scientists and engineers interested in learning about the current state of the art in radiation detectors and their applications in medical imaging.

Osama Mawlawi
University of Texas M.D. Anderson Cancer Center
Houston, Texas

COPYRIGHT (C) 2008 by the Society of Nuclear Medicine, Inc. DOI: 10.2967/jnumed.108.051318 